\title{
Electrical resistivity of single crystal arsenic at very low temperatures
}

\author{
C Uher and D T Morelli† \\ Department of Physics. The University of Michigan. Ann Arbor. MI 48109-1120. USA
}

Received 12 February 1986

\begin{abstract}
We have carried out high-precision measurements of the electrical resistivity on very high-quality arsenic single crystals along the binary direction below $4 \mathrm{~K}$. The results show that $\rho \sim 7^{n}$ with $n$ increasing from 3 to 4 below $2 \mathrm{~K}$. This strong temperature dependence is a signal of carrier-phonon scattering in this temperature regime. No evidence of a superconducting transition was observed down to as low as $15 \mathrm{mK}$ on our samples.
\end{abstract}

Previous studies of the electrical resistivity of arsenic along the binary axis have shown an interesting and as yet unexplained temperature dependence at low temperatures. Heremans et al (1977) measured $\rho_{11}$ using a standard four-probe technique on a single crystal grown by Jeavons and Saunders (1968) and found that in the range 4-30 K the resistivity behaved as $T^{3}$. Uher (1978) made measurements on a sample of the same origin and found that the cubic dependence continued down to at least $0.1 \mathrm{~K}$. While Bloch's theory predicts a crossover from a $T^{5}$ to a linear temperature dependence of the resistivity as temperature increases. and therefore gives $\rho \sim T^{3}$ over a limited temperature range, a cubic dependence extending over more than two decades of temperature is quite unusual. Furthermore, it is unlikely that such behaviour is associated with carrier-carrier scattering as this invariably leads to a $T^{2}$ rather than $T^{3}$ power law as shown by Baber (1937). Appel (1962) and more recently by Kukkonen and Maldague (1976).

As part of our research programme into the transport properties of semimetals, we have decided to look more closely into the mechanism of carrier scattering in arsenic. This decision was influenced by the availability of single crystals of arsenic of quality far superior to those used in previous studies. The aim of these experiments was to ascertain the limiting exponent of the temperature dependence of the resistivity as $T \rightarrow 0$ and to investigate the possibility of a superconducting transition in arsenic at very low temperatures.

Single crystals of arsenic were prepared from the melt by a procedure described in detail by Uher (1983). For our present purpose we simply note that this sample preparation technique produced arsenic specimens of unsurpassed quality as is attested by their very large residual resistance ratio, RRR. In this investigation we have used crystals with RRRS of 710, 2700 and approximately 9000: resistivity measurements described in the

† Present address: Physics Department. General Motors Research Laboratories, Warren, Michigan 480909055 . USA. 
Table 1. Transport parameters of arsenic single crystals.

\begin{tabular}{llrl}
\hline Designation & $\begin{array}{l}\text { Dimensions } \\
\left(\mathrm{mm}^{3}\right)\end{array}$ & RRR & $\begin{array}{l}\rho_{0} \\
\left(10^{-8} \Omega \mathrm{cm}\right)\end{array}$ \\
\hline A & $9.0 \times 1.5 \times 0.66$ & 708 & 3.80 \\
A2 & $11.0 \times 2.8 \times 1.6$ & 2700 & 0.93 \\
A. & $8.9 \times 2.7 \times 2.5$ & 9000 & 0.14 \\
\hline
\end{tabular}

literature until now have used samples with RRRS not exceeding 850 . The substantially greater purity of our samples is crucial for making accurate measurements of the resistivity at very low temperatures. since the impurity resistivity then represents a smaller fraction of

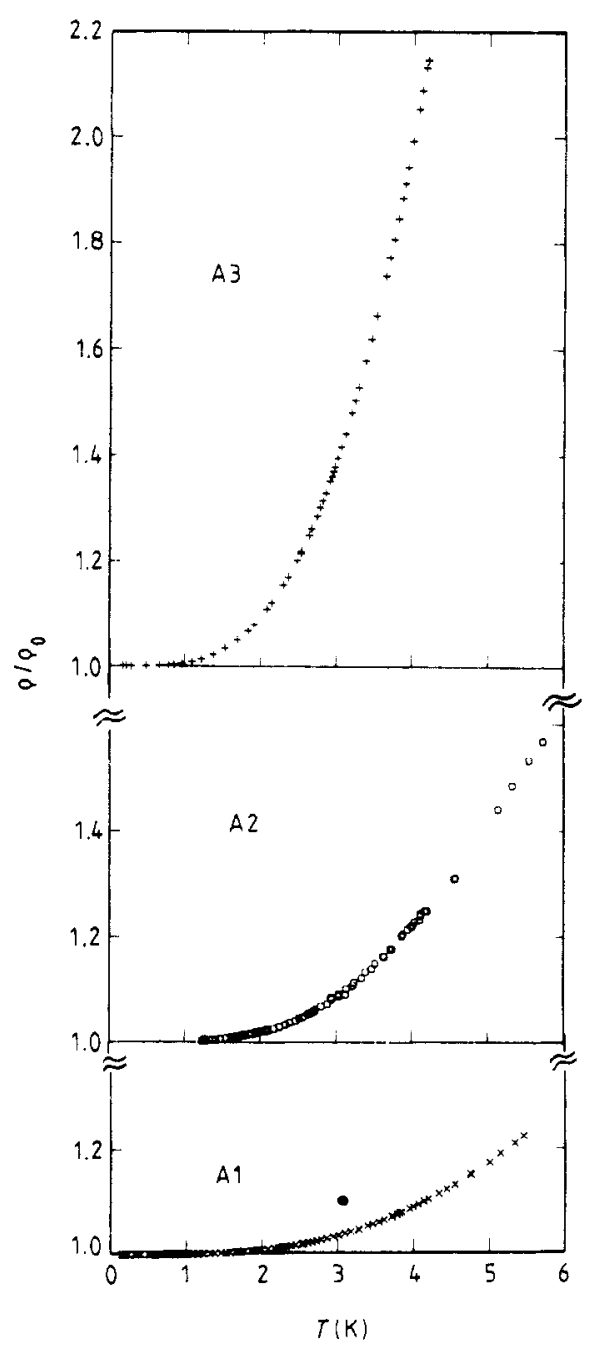

Figure 1. Resistivity of three samples of single crystal arsenic. A1, A2 and A3. The samples are designated according to table 1. 
the total electrical resistivity. As a consequence, the temperature-dependent term for higher quality samples is fractionally larger and easier to detect.

Sample preparation and mounting proceeded as follows: the specimen was removed from a vacuum dessicator and small arms were cut by spark erosion into its sides. The sample was then etched in a solution of two parts hydrofluoric acid to one part nitric acid to remove surface damage and oxidation introduced in the cutting process. Electrical contacts to the sample were made with 0.005 in $(0.125 \mathrm{~mm})$ diameter $\mathrm{Nb}-\mathrm{Ti}$ wires which were soldered with Wood's metal alloy using a tiny drop of mildly acidic flux. The crystal was then laid flat and tied with dental floss onto a copper bar which was bolted to the mixing chamber of a dilution refrigerator. Cigarette paper and Apiezon grease spread between the mating surfaces ensured that the sample was electrically insulated from, but thermally grounded to, the copper bar. This method of mounting was used as it was vibrationally and thermally much more stable than simply suspending the sample from the cryostat. Sample characteristics are given in table 1.

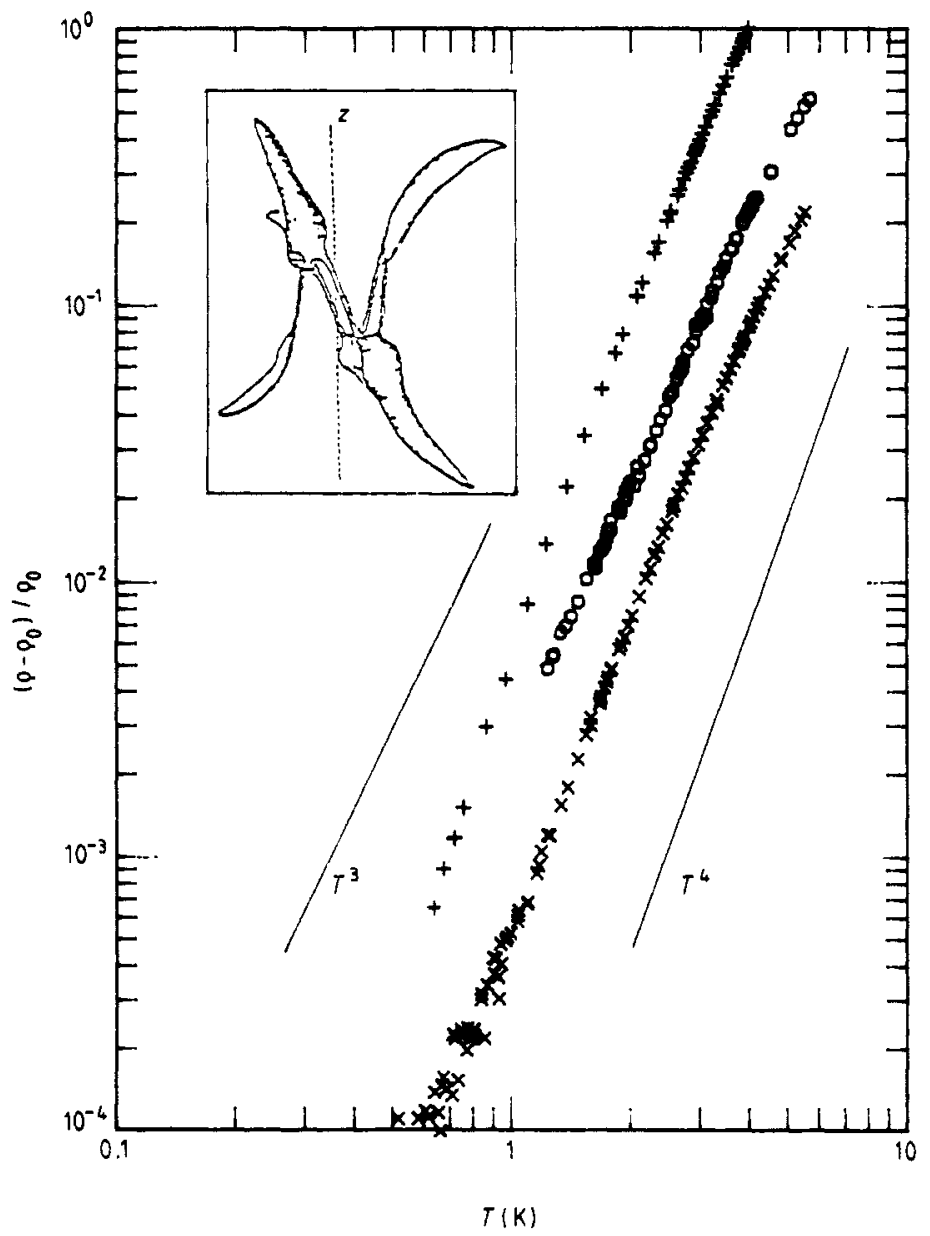

Figure 2. $\log -\log$ plot of the temperature-dependent part of resistivity. Samples are designated by the same symbois as in figure 1. In the insert is shown the hole Fermi surface of arsenic. 
The sample resistance was measured using a SQLID operating as a sensitive voltage null detector in a bridge circuit similar to that of Rowland and Woods (1976). In the particular case of arsenic the sensitivity of the method was limited by the magnitude of the sample resistance. The layered nature of the arsenic structure and the ease with which it can cleave placed rather stringent limitations on the length and cross-sectional area of the samples. Typical sizes were $10 \mathrm{~mm} \times 2 \mathrm{~mm} \times 1 \mathrm{~mm}$. As a result. all of our samples had resistances of a few microohms or less. Assuming that the ultimate sensitivity of the SQUID is about $10^{-14}$ volts. we could therefore achieve an accuracy of 'only about one part in $10^{5}$ using a current of $1 \mathrm{~mA}$. Higher sample currents would of course yield higher sensitivity but were found to produce self-magnetoresistance effects. The sample temperature was determined to within $0.5 \mathrm{mK}$ by a germanium resistance thermometer mounted next to the sample.

Figure 1 shows the resistivity of three arsenic samples. Difficulties with the dilution refrigerator prevented extension of the data for sample $\mathrm{A} 2$ to below $1.2 \mathrm{~K}$. For the other two samples. A 1 and A3, the resistivity is constant $\mathrm{u}$ ithin the precision of the measurement up to $0.4 \mathrm{~K}$ and then begins to rise very rapidly. We assume that Matthiessen's rule applies, so we can write

$$
\rho=p_{0}+\rho(T)
$$

where $\rho_{0}$ is the (constant) resistivity due to defect and impurity scattering and $\rho(T)$ is the temperature-dependent resistivity. From figure 1, $\rho_{0}$ is determined by extrapolating the curves to $T=0$ : the results are given in table 1 . The temperature-dependent resistivity is determined by subtracting $\rho_{0}$ from the measured resistivity at each point. Figure 2 shows the temperature-dependent resistivity $\rho(T)$ and. for greater clarity. in figure 3 we plot the temperature-dependent resistivity divided by $T^{4}$. From these plots it is quite clear that the resistivity begins to vary faster than $T^{3}$ below $2 \mathrm{~K}$, and in fact approaches a quartic temperature dependence at the lowest temperatures. This very rapid decline in $\rho(T)$ must be taken as a sign of the dominance of scattering of the carriers by lattice vibrations. The steady evolution of the temperature dependence of the resistivity to higher and higher powers of $T$ as the temperature is lowered suggests that the data could possibly be fitted by a Bloch-Grüneisen-type formula. Unfortunately. the Fermi surface of arsenic is so convoluted (see the inset of figure 2) that such a fitting procedure is probably not justified $\dagger$. Suffice it to say the dominant scattering of charge carriers in this system is by lattice vibrations. The resistivity data presented here provide further evidence, together with thermal conductivity and thermoelectric power studies of Morelli and Uher (1982), that the transport properties of arsenic are dominated by the carrier-phonon interaction.

Finally, we add that no evidence of a superconducting transition was detected down to $15 \mathrm{mK}$. We suggest that the slight fall-off which was observed below $0.1 \mathrm{~K}$ by Uher (1978) was due to the onset of superconductivity in the regions near the voltage contacts where some alloying of the arsenic and the (superconducting) solder may have occurred. This problem was circumvented in the present measurements by removing the voltage probes from the direct path of current flow via the small side arms.

The work was supported in part by the National Science Foundations Low Temperature Grant No DMR-8508392.

\footnotetext{
+ The best fit that we have obtained with the Bloch-Grüneisen formula suggests the need for a small $T^{2}$ term at the lowest temperatures (Morelli 1985). Whether this small quadratic contribution arises as a consequence of attempting to force the spherically symmetrical Fermi surface onto a highly convoluted Fermi surface of arsenic or whether it is real and perhaps implies a small carrier-carrier contribution is difficult to resolve.
} 


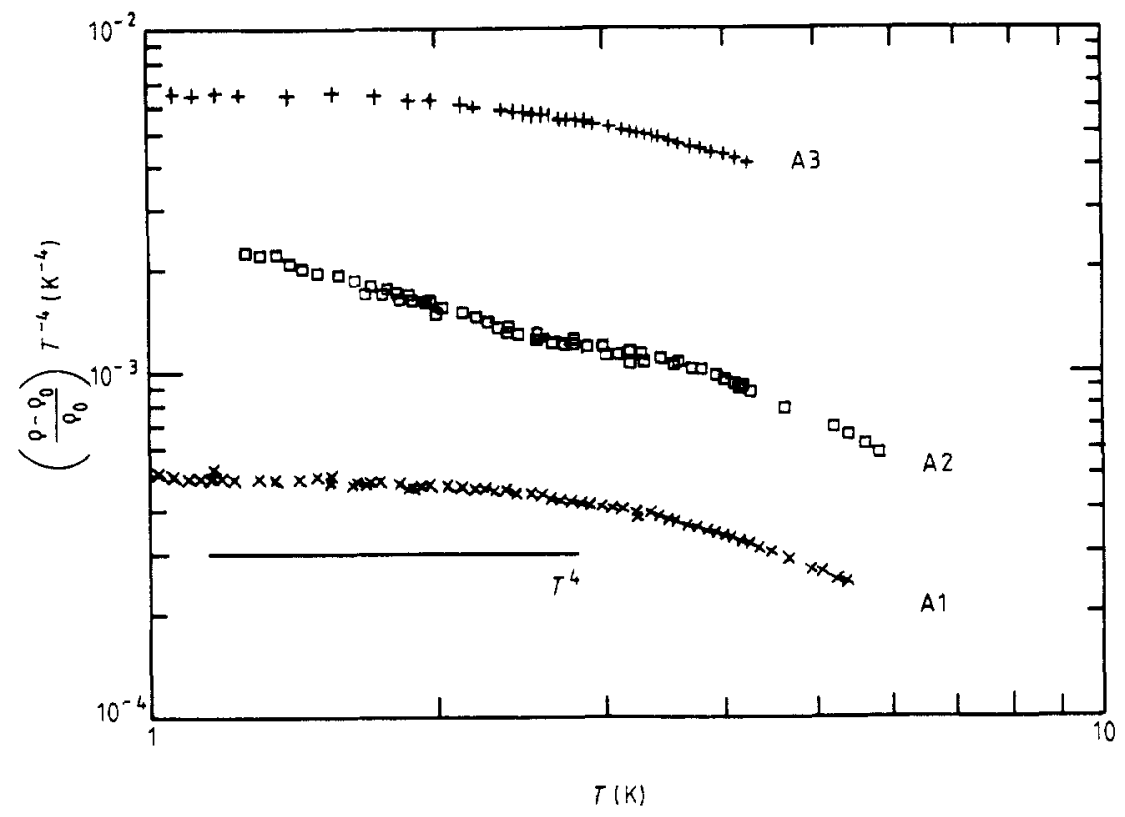

Figure 3. Temperature-dependent part of the resistivity plotted as $T^{-4} \Delta \rho / \rho_{0}$ against $T$ to highlight the quartic temperature dependence at the lowest temperature.

\section{References}

Appel J 1962 Phis. Rer. 1251815

Baber W G 1937 Proc. R. Soc. A 158383

Heremans J. Issi J-P. Rashid A A M and Saunders G A 1977 J. Phi's. C: Solid State Phi's. 104511

Jeavons A P and Saunders G A 1968 Br.J. Appl. Ph's. 1869

Kukkonen C A and Maldague D F 1976 J. Phi's. F: Mel. Phy's. 6 L301

Morelli D T and 1985 PhD Thesis University of Michigan

Morelli D T and Uher C 1982 Phis. Rer. B 266349

Rowland J A and Woods S B 1976 Rel. Sci. Itistrum. 47795

Uher C 1978 J. Phi'sique Coll. Suppl. 839 C6 1054

- 1983 J. Crisial Growth 62141 